\title{
Ciclo de Mejora en la asignatura Estadística Avanzada
}

\author{
Improvement Cycle in \\ Advanced Statistics course
}

LUIS ANDRÉS ZAMBRANA

ORCID: https://orcid.org/0000-0003-3606-7816

Universidad de Sevilla

Departamento de Economía Aplicada II

lazambrana@us.es

Fecha de recepción:

Fecha de aceptación:

DOI: http://dx.doi.org/10.12795/9788447221912.075

Pp.: 1731-1752 


\section{Resumen}

En esta comunicación describiré la aplicación de un Ciclo de Mejora en el Aula (CIMA) en la asignatura de Estadística Avanzada, basado en el planteamiento de un caso real al que los estudiantes han aplicado las herramientas de Inferencia Estadística. Para ello han recorrido el proceso que debería seguir cualquier investigador que, partiendo del interés en conocer una realidad cuya información no se encuentre registrada en publicaciones o estadísticas oficiales, ha de diseñar la forma de recoger información, poner en práctica el procedimiento elegido y aplicar las herramientas de las que nos dota la inferencia estadística para, finalmente, llegar a conclusiones numéricas sobre la realidad objeto de interés. La realidad sobre la que han trabajado está ligada a la situación de Emergencia Climática, con lo que el ciclo ha perseguido un doble objetivo, enseñar con una metodología basada en problemas y educar en valores.

Palabras claves: Estadística Avanzada; Aprendizaje en Disciplinas Técnicas; Grados en Administración y Dirección de Empresas, Investigación y Técnicas de Mercado y Economía. Docencia Universitaria; Experimentación Docente Universitaria; Educación en Valores.

\section{Abstract}

This paper describes the application of a cycle of instructional improvement within a course of Advanced Statistics, based on the proposal of a real case study to the students for them to apply tools of statistical inference. With this purpose they had to follow the sequence that any researcher would follow, starting from the interest in reaching some information of a fact which is not registered in oficial publications or statistic reports, then designing the plan for data gathering, implementing it and applying the statistical inference tools to finally achieve numerical conclusions regarding the subject of study. The field of the study case is like de to Climate Emergency, what extend this cycle to a dual aim: teaching using a problems based methodology and educating in values.

Keywords:Advanced Statistics; Learning in Technical Disciplines; Degrees in Business Administration and Management; Research and Market and Economics Techniques; University Teaching; ; University Teaching Experimentation; Education in Values. 


\section{Descripción del contexto de la intervención}

Este CIMA lo he llevado a la práctica en la asignatura Estadística Avanzada, de segundo curso del Grado en Administración y Dirección de Empresas, ha sido implementado en los tres grupos impartidos durante el curso 2019/2020 (de los ocho existentes). Se trata de una asignatura con seis créditos (60 horas presenciales), repartidos en 30 bloques de 2 horas, 2 por semana.

Entre los tres grupos suman un total de 230 estudiantes matriculados (81, 82 y 67 respectivamente), de los que 88 son repetidores (38\%), aunque solo 30 (13\%) se han presentado a alguna convocatoria anterior. Este curso es el segundo en el que desarrollo un CIMA, con lo que los estudiantes ya vienen con información previa, más allá de lo que pueda recoger el proyecto docente. A mi entender es importante la trasmisión de información entre ellos, pues creo que esta es una de las razones que puede explicar el aumento significativo de presencialidad, mientras que en el curso anterior (2018/2019) la asistencia a clase se situaba en un número cercano a los 40 estudiantes (Andrés, 2019), este año está por encima de los 60. Las aulas en las que tienen lugar las clases tienen las bancas y pupitres fijos, con una disposición pensada, exclusivamente, para las clases magistrales, no aptas para el trabajo en grupo de los estudiantes, que eligen sus puestos sin patrón alguno y que tiene como consecuencia una alta dispersión en el espacio, es curioso que la primera fila, la más cercana a la pizarra, suele estar vacía, lo que denota, sin duda, la lejanía a lo que alli va a ocurrir.

El contenido de la asignatura parte de la Teoría de la Probabilidad y va recorriendo las herramientas que ésta desarrolla para llegar hasta la Inferencia Estadística., si la consideramos en grandes bloques, serían tres, a saber: Teoría de la Probabilidad y Variables Aleatorias, Teoría de Muestras e Inferencia Estadística (Estimación y Contrastes 
de Hipótesis) (Cano, 2018). La asignatura es una de más dificiles de superar para los estudiantes del grado, como muestra el hecho de que cuente con un porcentaje alrededor al $40 \%$ de repetidores.

Dado que el Cima comprende la asignatura completa y este artículo se cierra cuando al curso le quedan aún 7 semanas, coincidencia de fechas y circunstancias que ya ocurrieron el pasado curso, incluiré los resultados anteriores enlazados con el desarrollo de este.

\section{Diseño previo del Ciclo de Mejora en el Aula}

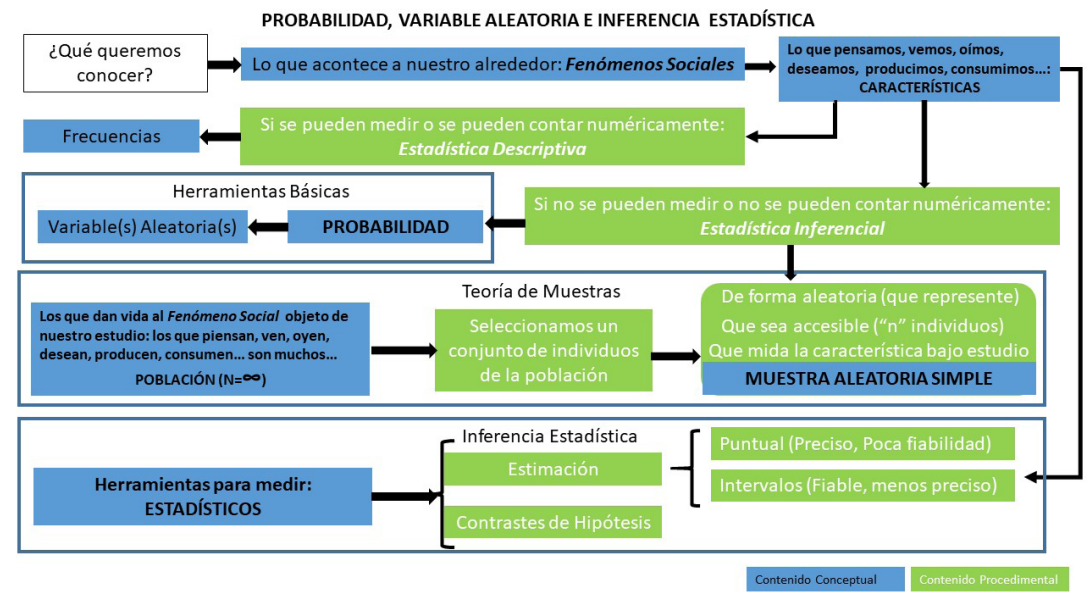

Figura 1. Mapa de contenidos para el proceso de Inferencia Estadística.

Partiremos de características que son objeto de nuestro interés, necesariamente ligada a un fenómeno social. En la medida en la que dicha característica no esté registrada, censada o descrita en lugar alguno, es el investigador el que ha de "construir" el conocimiento de la misma mediante procedimientos inferenciales. En esta asignatura el procedimiento se cimenta sobre la Teoría de la Probabilidad y su aplicación en Variables Aleatorias. El camino a la inferencia, sobre la base antes mencionada, se construye 
a partir de una Muestra Aleatoria Simple sobre la que definiremos Estadísticos que usaremos para Estimar y/o Contrastar Hipótesis, procedimientos ambos que definen el objeto último de la asignatura.

\section{Modelo metodológico}

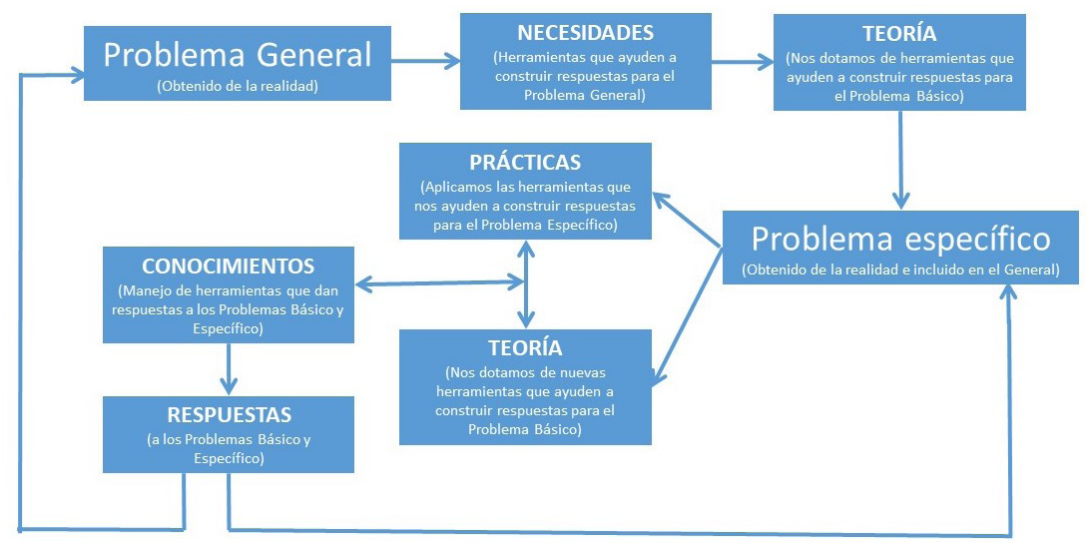

Figura 2. Modelo metodológico.

Como modelo metodológico reproduzco el que ya usara en el CIMA del curso anterior (Andrés, 2018) puesto que dio respuesta más que satisfactorias desde el punto de vista de las actividades de contrastes y, sobre todo, de los resultados en las evaluaciones oficiales de los estudiantes como comentaré más adelante en esta publicación. Hasta la puesta en práctica de la educación basada en problemas reales, mi docencia transitaba un camino que buscaba proveer a los estudiantes de soluciones a problemas que ellos nunca se habían planteado, lo que dificultaba en gran medida la aprehensión por su parte de las herramientas que pretendía trasmitirles. Arrancar desde un problema real, cercano, que forme parte del contexto en el que viven y en el que se mueven, facilita atrapar su atención y, sobre todo, despertar sus expectativas a la hora de solucionarlo (Paenza, 2014). 
A la estrategia docente que supone enseñar basándonos en problemas reales le añadimos el tercero de los principios establecidos en el preámbulo de la "Magna Charta Universitatum", firmada en Bolonia en 1988 y que ha configurado, para bien o para mal, la actual estructura universitaria, a saber "la universidad debe asegurar a las futuras generaciones la educación y la formación necesarias que contribuyan al respeto de los grandes equilibrios del entorno natural y de la vida." (Bolonia 1988). En este sentido mi papel de formador me exige habilitar y capacitar a los estudiantes en el uso de las herramientas de Inferencia Estadistica y fomentar una mirada crítica y comprometida con la sociedad que les provea de una educación universitaria pública (no debe entenderse esta afirmación como la negación de dicho papel a instituciones privadas, pero en el caso de la pública es un deber) (Manzano, 2012)

Previo al inicio de curso un grupo de docentes de distintas disciplinas hemos coincidido en la necesidad de abordar en nuestras aulas la actual crisis climática. Aprovechando todo el movimiento de concienciación y, por qué no decirlo mediático, generado alrededor de las cumbres, conferencias, rondas...que se están sucediendo, así como las adhesiones de instituciones cercanas a la declaraciones de emergencia climática, en nuestro caso el Consejo de Gobierno de la Universidad y el Ayuntamiento, ambos de Sevilla, son caldo de cultivo propicio para enfocar la mirada hacia lo que nos está pasando e intentar usar los contenidos de nuestras materias al servicio de un enfoque científico de dicha mirada. Para ello hemos elaborado materiales de forma colaborativa con los que arrancar el curso y que describiré en la secuencia de las clases.

El objetivo final del curso es cuantificar aspectos de la percepción de emergencia climática que tienen los ciudadanos, asignando valores a parámetros desconocidos de algunas de las características que definen dicha percepción, y esta propuesta nos llevará al planteamiento del 
problema específico ¿Somos consciente los ciudadanos que vivimos un momento de crisis climática? El desarrollo del ciclo de mejora se diseña como un proceso real encaminado a dar respuesta a dicha pregunta con números reales (Finkel 2008).

El proceso nos demandará herramientas de las que nos dotaremos en clases de teoría y requerirá un trabajo de campo que realizarán los estudiantes fuera del aula. Con el resultado obtenido aplicaremos, en sesiones prácticas, las herramientas de las que nos hemos dotado en teoría.

\section{Secuencia de actividades programada}

\begin{tabular}{|c|c|}
\hline SEMANA & CONTENIDO \\
\hline \multirow{3}{*}{$\begin{array}{c}\text { Semana } 1 \\
\text { (23-27/Septiembre) } \\
4 \text { horas }\end{array}$} & $\begin{array}{l}\text { Fase Metodológica: Presentación y Planteamiento del } \\
\text { Problema para trabajar }\end{array}$ \\
\hline & $\begin{array}{l}\text { Presentación de la asignatura, con base en dos pilares } \\
\text { sobre los que desarrollaremos los contenidos de la misma. } \\
\text { 1.- El planteamiento de la misma en base a construir la } \\
\text { respuesta a un problema real. } \\
\text { 2.- El problema real al que daremos respuesta saldrá } \\
\text { del contexto de emergencia climática en el que nos } \\
\text { encontramos. } \\
\text { Se pone a disposición de los estudiantes lectura y enlaces } \\
\text { sobre “emergencia climática", así como la declaración } \\
\text { aprobadas por las instituciones cercanas, a saber, } \\
\text { Universidad de Sevilla y Ayuntamiento de Sevilla. } \\
\text { Trabajo en grupo y puesta en común: ¿Cómo percibo la } \\
\text { situación actual en relación a la "emergencia o crisis } \\
\text { climática"? }\end{array}$ \\
\hline & Recursos: Documentación y lecturas en Enseñanza Virtual. \\
\hline $\begin{array}{c}\text { Semana } 2 \\
\text { (30/Septiembre-2/ } \\
\text { Octubre) } \\
4 \text { horas }\end{array}$ & Fase Metodológica: Teórico-Práctica \\
\hline
\end{tabular}

Jornadas de Formación e Innovación Docente del Profesorado | № 2 (2019) Esta obra se distribuye con la licencia Creative Commons Reconocimiento-NoComercial-SinObraDerivada Internacional (CC BY-NC-ND 4.0.) 
Propuesta de trabajo individual: ¿Qué fenómenos relacionados con la "emergencia climática" podrían ser objeto de estudio para un economista? ¿y de trabajo con las herramientas que nos provee la Estadística Inferencial? (Pregunta a usar en contraste, Actividad 01) Se habilita una dirección de correo electrónico para que, tras la sesión de trabajo y discusión en clase, elaboren una propuesta por escrito y la envíen para trabajar en la siguiente clase.

Con las propuestas por ellos formuladas, analizamos ¿qué es objeto de estudio de los economistas? y ¿qué podemos trabajar con las herramientas de la Estadística Avanzada

Recursos: Clase hablada y Power Point con resumen del trabajo de los estudiantes.

Fase Metodológica: Teórico

Trabajamos las características que deben tener los fenómenos objeto de nuestro trabajo. Comparamos los resultados de las elecciones generales (04/2019) con el último informe del CIS referido a la misma realidad (07/2019), al objeto de ilustrar las diferencias entre describir

Semana 3 (Estadística descriptiva) e inferir (Estadística inferencial):

(7-11/Octubre) Trabajamos la Teoría de la Probabilidad y Variables 4 horas Aleatorias y enviarlas a la dirección habilitada (Actividad 02, para el contraste).

Trabajo individual: Tienen que proponer tres realidades ligadas al cambio climático susceptible de trabajar con variables aleatorias y determinar todos los parámetros de las mismas.

Recursos: Clase hablada y búsqueda en la Web.

Semana 4

(14-18/Octubre) Fase Metodológica: Problema Real/ Problemas Específicos 4 horas 
A partir de las tres realidades propuestas por los estudiantes, elaboramos un listado/ranking y trabajamos en clase la viabilidad de abordar, con los recursos de los que disponemos, el estudio de las mismas. tras la reflexión colectiva y analizar la viabilidad, vuelven a proponer, en este caso una sola realidad (Actividad 03, para el contraste) y una propuesta más elaborada.

Seleccionamos una realidad para trabajar una variable Aleatoria Discreta, abordaremos el conocimiento de "la proporción de la ciudadanía que percibe que vivimos en una situación de emergencia climática". Y seleccionamos el conocimiento de la "cantidad de residuos que reciclamos los ciudadanos" como realidad a abordar desde una Variable Aleatroia Continua.

Recursos: Clase hablada y Power Point con los resultados de sus trabajos.

Fase Metodológica: Teoría y Prácticas

Para poder trabajar las realidades seleccionadas, es decir, "la proporción de la ciudadanía que percibe que vivimos en una situación de emergencia climática" y la "cantidad de residuos que reciclamos los ciudadanos" construimos en la clase las distribuciones de Bernouilli y Normal.

Para la Bernouilli realizamos un experimento en la propia clase en base a la forma en la que se desplazan desde su domicilio a la Facultad. Para la distribución Normal

Semana 5 (21-25/Octubre) 4 horas conduzco el proceso para que ellos elaboren la campana de Gauss a partir de su percepción de la altura de las personas.

Finalmente formalizamos las distribuciones con el instrumental que necesitaremos, fundamentalmente un manual de Estadística con las tablas de cálculo que ha elaborado un compañero del Dpto. enfocado a los contenidos de la asignatura.

Se propone un ejercicio de manejo de las tablas (Actividad 05 para el contraste)

Recursos: Encuesta en la clase, trabajo colectivo dirigido por el profesor y manual-tablas estadísticas adaptado. 
Fase Metodológica: Prácticas

Trabajo en clase con distribuciones normales. Busco información sobre el consumo de energía electrica de los habitantes de Sevilla y construimos una distribución Normal a partir de dicha información.

Semana 6

Con la distribución que hemos construido elaboran, de (28/Octubre) forma indicvidual, un informe respondiendo a cuestiones relativas al consumo de electricidad de los sevillanos 2 horas (Actividad 04, para el contraste). El objeto principal de esta actividad es que sepan el significado de lo que contienen las tablas, que no se convierta en una búsqueda mecánica y que sepan "leer" en los números contenidos en dichas tablas.

Recursos: Búsqueda de información en la Web y manejo de tablas estadísticas.

Fase Metodológica: Prácticas

La primera parte la dedicamos a corregir la actividad 04, y una vez finalizada y sin previo aviso, les entrego un informe sobre el consumo de energía eléctrica en España y unas cuestiones a trabajar sobre el mismo. Disponen del resto de la clase (aprox. 45 minutos) para elaborarlo y

Semana 7

(4 a $8 /$ Noviembre) 4 horas pueden usar todos los recursos que consideren oportunos, incluso consultar entre compañeros (Actividad 05 para el contraste). Al final de la clase me entregan el informe que les repartí con sus aportaciones. Y en la enseñanza virtual les dejo el mismo informe para que, en esta ocasión, lo hagan en sus casas y lo entreguen la siguiente semana (Actividad 06 para el contraste).

Recursos: Búsqueda de información en la Web y trabajo en clase.

Semana 8

(11 a 15/ Noviembre) 4 horas 


\begin{tabular}{|c|c|}
\hline & $\begin{array}{l}\text { Explicación del vector aleatorio y sus aplicaciones en } \\
\text { nuestro trabajo, a saber, Teorema Central del Límite y } \\
\text { Distribución Normal Multivariante. } \\
\text { Prácticas en el aula con ejemplos reales. } \\
\text { Para el vector normal multivariante trabajamos el consumo } \\
\text { de electricidad en los hogares por distintas agrupaciones: } \\
\text { Electrodomésticos, Iluminación y Térmicos. Les dejo la } \\
\text { información en la Enseñanza Virtual y ellos lo trabajan en } \\
\text { casa y lo entregan la siguiente semana (Actividad } 07 \text { para } \\
\text { el contraste). }\end{array}$ \\
\hline & $\begin{array}{l}\text { Recursos: Clase Magistral, búsqueda de información en la } \\
\text { Web y trabajo en clase. }\end{array}$ \\
\hline \multirow{3}{*}{$\begin{array}{c}\text { Semana } 9 \\
\text { (18 a 22/ Noviembre) } \\
4 \text { horas }\end{array}$} & Fase Metodológica: Practica \\
\hline & $\begin{array}{l}\text { Prácticas en el aula con ejemplos reales. } \\
\text { Para el vector normal multivariante y el teorema central del } \\
\text { límite trabajamos información proporcionada por el Plan } \\
\text { Integral de la Bicicleta de la Universidad de Sevilla (PIBUS) } \\
\text { y el Informe de } 2018 \text { de ECOEMBES. Los datos extraídos de } \\
\text { ambos informes se los pongo disponibles en la Enseñanza } \\
\text { Virtual, parte lo trabajamos en clase y el resto lo trabajan } \\
\text { en casa (Actividad } 08 \text { para el contraste). } \\
\text { Esta semana termina el primer bloque de la asignatura, } \\
\text { alq ue podemos llamar Herramientas Básicas. Les dejo } \\
\text { en Enseñanza Virtual un documento para que puedan } \\
\text { practicar y fijamos el día } 2 \text { de diciembre para la realización } \\
\text { de una prueba en la que se les pedirá que manejen las } \\
\text { mismas sobre la base de un caso real. }\end{array}$ \\
\hline & $\begin{array}{l}\text { Recursos: Información de la Web, trabajo en clase y trabajo } \\
\text { en casa. }\end{array}$ \\
\hline $\begin{array}{c}\text { Semana } 10 \\
(25 \text { a } 29 / \text { Noviembre }) \\
4 \text { horas }\end{array}$ & Fase Metodológica: Problema Real \\
\hline
\end{tabular}

Jornadas de Formación e Innovación Docente del Profesorado | № 2 (2019) Esta obra se distribuye con la licencia Creative Commons 


\begin{tabular}{|c|c|}
\hline & $\begin{array}{l}\text { Definimos con precisión la característica a trabajar en el } \\
\text { conjunto de los tes grupos en los que se ha puesto en } \\
\text { marcha el ciclo. } \\
\text { Variable: "Precepción que tiene los estudiantes de la } \\
\text { Universidad de Sevilla sobre la situación de Emergencia } \\
\text { Climática” (Es una distribución de Bernouilli, en la que } \\
\text { intentaremos estimar la proporción de estudiantes que } \\
\text { percibe la situación de emergencia climática). } \\
\text { Iniciamos el camino de Inferencia Estadística. En esta } \\
\text { semana trabajamos la Muestra Aleatoria Simple, los } \\
\text { Estadísticos y su funcionamiento en nuestro contexto de } \\
\text { trabajo. }\end{array}$ \\
\hline & Recursos: Taller conceptual y prácticas \\
\hline \multirow{3}{*}{$\begin{array}{c}\text { Semanas } 11 \text { a } 15 \\
\text { (2 de diciembre a } 17 \\
\text { de enero) } \\
20 \text { horas }\end{array}$} & Fase Metodológica: Problema real y soluciones al mismo \\
\hline & $\begin{array}{l}\text { En las } 4 \text { últimas semanas del curso, la clase se convierte } \\
\text { un taller de trabajo. Los estudiantes, con las herramientas } \\
\text { aprendidas en el primer bloque temático de la asignatura } \\
\text { y las indicaciones del profesor, construyen el andamiaje de } \\
\text { la Inferencia Estadística. } \\
\text { 1.- Muestra Aleatoria Simple, 2.- Estadísticos, } \\
\text { 3.- Estimadores. } \\
\text { Realizan una encuesta, cada estudiante se la pasa a } 10 \\
\text { individuos, intentado mantener la independencia entre los } \\
\text { entrevistados. } \\
\text { Tratamos los datos en clase. } \\
\text { Explotamos los resultados y finalmente hacemos una } \\
\text { presentación de los mismos. }\end{array}$ \\
\hline & Recursos: Taller conceptual, trabajo de campo y prácticas \\
\hline
\end{tabular}

Las clases se inician con un doble objetivo, por un lado, que esté presente el mayor número de aspectos posibles de la situación de crisis climática en la que estamos sumidos, para lo que se ha dispuesto en la enseñanza virtual un documento con cuestiones previas y respuestas de científicos, expertos, comunicadores... obtenidas de artículos, videos, noticias... Por otro lado, trabajar sobre un problema real y de total actualidad (el comienzo de las clases ha coincidido con la semana por el clima que NN.UU. celebró en New York el 23 de septiembre de 2019). 
Tras presentar el programa de la asignatura, remarcando la necesidad de la implicación de los estudiantes para que funcione el método, les justifico la elección de "la emergencia climática" como problema real alrededor del cual construiremos la asignatura.

La primera clase la ocupamos por completo con la reflexión, el debate y la puesta en común sobre lo que percibimos en referencia a la situación del planeta y las consecuencias de nuestro modo de vida. Los estudiantes forman grupos de 4 y durante 15 minutos debaten en el seno de cada grupo cómo perciben la situación actual del planeta. Pasado ese tiempo un portavoz de cada grupo cuenta al resto de la clase las conclusiones que han obtenido, una vez expuestas todas las conclusiones intentamos consensuar una respuesta a la pregunta ¿Cómo percibe el conjunto de la clase la situación de emergencia climática?: "Vivimos una situación de emergencia en relación al deterioro del planeta con graves consecuencias en un corto plazo. La mayor parte de la responsabilidad recae en las grandes empresas y los gobiernos que no legislan para reducir los efectos de las actividades de las primeras. Los ciudadanos somos responsables por nuestro modo de vida, pero somos insignificantes a la hora de revertir la situación"

Los estudiantes proponen qué aspectos de "Emergencia Climática" les gustaría que construyéramos conocimiento a partir de las herramientas que nos proporciona la inferencia estadística y que esté en el ámbito de "lo social". Dedicamos una clase a debatir sus propuestas y centrar lo que será nuestro problema real, llegamos al final al siguiente acuerdo:

- Problema real a trabajar, poner número al siguiente planteamiento: "Proporción de ciudadanía que percibe que vivimos un momento de emergencia climática". Elegimos este problema por su simplicidad a la hora de ser abordado (el ciudadano lo percibe o no 
lo percibe) permitiéndonos desplegar todo el instrumental de la disciplina.

- A la vez que construimos la respuesta al problema real hicimos incursiones en problemas más complejos, que requieren el mismo instrumental, pero sobre los que no disponemos de medios y recursos para construir una respuesta real, por ejemplo (sacado de las propuestas de los estudiantes) "¿Qué cantidad de plásticos consumimos?" o "¿Qué cantidad de plásticos reciclamos?

- A clase llevo cuestiones reales ligadas a la situación de emergencia climática: consumo de energía según informe de NN.UU; modo de transporte de los estudiantes de la US según informe del Plan Integral de la Bicicleta de la US, reciclaje de residuos de los españoles del informe de ECOEMBES... Toda esta información se despliega con un doble objetivo, por un lado hablar de las cuestiones de fondo, es decir, de "lo que hacemos" en relación a nuestra contribución al cambio climático y por otro usar las herramientas de Estadística Avanzada.

- Para las clases prácticas, es decir para el uso del instrumental propio de la asignatura, las distribuciones de probabilidad en general y de la distribución Normal y Binomial en particular, en este ciclo he puesto en marcha una experiencia que a mi entender ha funcionado bien. Al principio de las clases les entregaba, sin previo aviso y puntuable, un folio con información real para que ellos la trabajaran y me lo entregarán, el hecho de que fuese puntuable les implicaba a tope en el trabajo y en el uso de las herramientas. En la plataforma de Enseñanza Virtual disponían de la misma actividad para que la trabajaran en casa y la entregaran por escrito en la siguiente clase, por supuesto también puntuable. El objeto de este proceder es que reflexionen sobre los errores y construyan su propio camino a la solución 
con el uso de las herramientas de la asignatura (Morin y col, 2002).

En la onceava semana del curso y, por tanto, de este CIMA, coincidiendo con la fecha de cierre y envío de esta comunicación, los estudiantes (aproximadamente 180 entre los tres grupos objeto del ciclo) están dando forma al trabajo que dará respuesta al problema real:

- ¿Qué estimarán?: "La proporción de estudiantes de la Universidad de Sevilla que perciben la situación actual cómo de Emergencia Climática". Han decidido hacer la estimación sobre la población "Estudiantes de la Universidad de Sevilla" por ser más cercana y accesible sin que ello afecte al uso de las herramientas objeto de la asignatura.

- ¿Cómo estimarán?: Cada estudiante hará 10 encuestas a estudiantes de la Universidad de Sevilla, para lo que diseñamos un reparto que se asemeje, en la medida de lo posible, a un reparto aleatorio. Cuando realicen la encuesta tendremos un trabajo de campo con, aproximadamente, 1800 encuestas para una población de alrededor de 60000, lo que, sin duda alguna, es una encuesta muy por encima de lo que sería un trabajo profesional.

- Durante el curso necesitaremos la asistencia técnica de otras disciplinas, a saber, habremos de precisar en qué consiste "percibir" o acotar en qué consiste una situación de "emergencia climática", para ello contaremos con la presencia (ya acordado) de estudiantes de la asignatura Psicometría (Grado de Psicología) del Prof. Vicente Manzano o con estudiantes de Climatología del Grado de Geografía de la Profa. Mónica Aguilar.

- Una vez tengamos los resultados procederemos a las estimaciones y a la elaboración de un informe final. Para lo que concerté con el decano de la Facultad habilitar un espacio (salón de Actos o de Grados) en el que hacer una presentación pública, así 
mismo hablé con la periodista de "El País" Ángeles Lucas para que le haga un hueco al trabajo de los estudiantes en su blog "Planeta Futuro". Con ello pretendo que los estudiantes sean conscientes de que con las herramientas que han usado y el trabajo realizado han creado conocimiento, han generado algo que no existía poniéndole números a realidades que nadie se las había puesto hasta este momento y que, a partir de ahora, si alguien quiere saber la proporción de estudiantes que son conscientes de la situación de Emergencia Climática encontrarán ese dato por qué ellos lo crearon y lo difundieron.

\section{Evaluación}

Para la evaluación del ciclo de mejora usaré la cuestión que les planteé encaminada a determinar un problema real sobre el que construir el conocimiento. Lo estudiantes me enviaron sus propuestas de trabajo para realizarlo con unas herramientas que para ellos son desconocidas. Al final de curso les plantearé una cuestión similar, a la que habrán de aplicar el arsenal de herramientas aprehendidas, comparando las respuestas en uno y otro punto estaremos en disposición de evaluar. El cuestionario final, además de servir para evaluar el ciclo, servirá para evaluar a los estudiantes.

\section{Cuestionario inicial:}

Proponga aspectos y/o características sociales cuyo conocimiento podamos abordar con el uso de la inferencia estadísticas.

Las propuestas se han movido en tres grandes bloques:

- Descripción de lo que significa Cambio Climático, Crisis Climática o Emergencia climática, las causas y las 
consecuencias. No son estrictamente propuestas, más bien respuesta a una hipotética sobre dichos conceptos. A lo sumo lo han acompañado de propuesta de actividades en el ámbito de movimientos sociales (reciclar más, consumir menos plásticos...)

- Propuestas que se moverían más en el objeto de estudio de otras materias y que escaparían a las capacidades de las ciencias sociales. Medir la subida de los mares, la subida de temperatura...

- Propuestas más enfocadas a lo que podemos hacer: medir la percepción de la situación, lo que reciclamos, lo que consumimos... En esta última centramos la sesión de trabajo para elegir el problema real sobre el que trabajar.

\section{Cuestionario Final:}

Asistimos a un momento que podemos calificar de Emergencia Climática. La Universidad de Sevilla asi lo ha declarado institucionalmente. Estamos interesados en conocer si los estudiantes de esta Universidad son conscientes de dicha situación de emergencia.

Para ello nos encargan que realicemos un estudio en el que intentaremos dar respuesta a la siguiente cuestión ¿Qué proporción de estudiantes de la US son conscientes de que vivimos en una situación de Emergencia?

1. ¿Qué variable aleatoria considera adecuada para poder estudiar dicha proporción? (Justifique su elección, defina la variable, establezca su recorrido, la distribución de probabilidad que sigue, los parámetros de la distribución y el significado de los mismos)

2. Una vez planteado el punto de partida con la variable a utilizar y su distribución, establezca el procedimiento que seguirá para estimar lo necesario en dicha distribución de probabilidad 
¿Qué herramientas estadísticas usarás? ¿Cómo funcionan y que propiedades tienen?

3. Hacemos una selección aleatoria de 1800 estudiantes, de los que 1260 dicen ser conscientes de la situación de emergencia. Haga la mejor estimación puntual posible de la proporción de estudiantes conscientes de la situación de emergencia, señale las propiedades que tiene, así como los inconvenientes que esta estimación plantea. Haga una estimación por intervalos de dicha proporción con una confianza del 95\% y coméntela en relación a la estimación puntual.

4. Con los datos obtenidos en la muestra de los 1800 estudiantes ¿A qué niveles de significación podríamos aceptar la afirmación de que "la mayoría de los estudiantes tienen conciencia de la situación de emergencia climática? Comente los resultados.

5. Para estudiar el comportamiento de los estudiantes de la US en relación al reciclaje de embalajes de plásticos ¿Qué supuestos deberiamos hacer para poder aplicar las herramientas aprendidas en Estadística Avanzada? Seleccionamos de forma aleatoria 100 estudiantes, obtenemos una cantidad media diaria de reciclaje de envases de plástico de 240 gramos, con una desviación estándar de 60 gramos. Estime un intervalo del 95\% de confianza para la cantidad media diaria de envases de plástico reciclado por los estudiantes de la US y a la vista de los resultados diga si es aceptable con un 5\% de confianza que la cantidad media de envases de plástico reciclados por los estudiantes de la US es de 250 gramos, razonando su respuesta ¿A qué nivel de significación podriamos aceptar que es superior a 250 gramos diarios por estudiante?

Jornadas de Formación e Innovación Docente del Profesorado | № 2 (2019) Esta obra se distribuye con la licencia Creative Commons Reconocimiento-NoComercial-SinObraDerivada 4.0 Internacional (CC BY-NC-ND 4.0.) 
Entre el cuestionario inicial, en el que pretendía acercarme a la idea que los estudiantes tenían de los contenidos de Estadística Avanzada y sus aplicaciones, en nuestro caso a la realidad ligada a la situación de Emergencia Climática, y el cuestionario final que será el examen que han de realizar como parte de su evaluación y, por tanto, de su calificación final, ellos han ido respondiendo actividades en las que buscado conocer cómo estaban aprehendiendo las herramientas de las que les iba dotando.

Este proceder no se ha basado en la escalera de aprendizaje estableciendo dos puntos de comparación con las mismas referencias y puedes medir los avances, como ya hiciera en el CIMA del curso anterior, para este curso estoy intentando medir, a través de las actividades y los trabajos, como van adquiriendo las capacidades y habilidades. Es mi intención que la prueba final, antes descrita, me permita cuantificar en qué medida adquirieron los conocimientos objeto de la asignatura.

\section{Evaluación del ciclo}

Como ya señalara anteriormente, esta comunicación la cierro sin terminar el curso, situación que se repitiera el pasado año (las comunicaciones se entregan en noviembre y el curso termina en enero del año siguiente), es por ello que aquí voy a utilizar los datos obtenidos en el ciclo anterior, comparando los resultados de dos grupos en los que puse en marcha CIMAs de asignatura completa, frente a un grupo que siguió la asignatura con una metodología tradicional.

La tabla 1 muestra la comparativa de los resultados obtenidos en los tres grupos que impartí el pasado curso de la asignatura Estadística Avanzada, en los grupos 1 y 3 apliqué un ciclo de mejora docente durante todo el curso. 
Mientras que, por circunstancias sobrevenidas, el grupo 5, lo impartí siguiendo la metodología tradicional, a saber, clases magistrales de teoría y desarrollo de casos prácticos explicados en la pizarra.

Tabla 1. Comparativa de resultados.

\begin{tabular}{|l|l|l|l|l|l|l|l|}
\hline & \multicolumn{2}{|l|}{ GRUPO 1 } & \multicolumn{2}{l|}{ GRUPO 3 } & \multicolumn{2}{l|}{ GRUPO 5 } \\
\hline & \multicolumn{2}{|l|l|l|l|l}{ № } & \% & № & \% & № & \% \\
\hline Matriculados & \multicolumn{2}{|l|}{71} & & \multicolumn{2}{l|}{72} & \multicolumn{2}{l|}{73} \\
\hline No Presentados & 28 & 39,4 & 19 & 26,4 & 50 & 68,5 \\
\hline Superaron la asignatura & 37 & 52,1 & 38 & 53,8 & 9 & 12,3 \\
\hline No superaron la asignatura & 6 & 8,5 & 15 & 20,8 & 14 & 19,2 \\
\hline Nota media & 6,24 & 5,58 & 2,83 & & & \\
\hline
\end{tabular}

Los tres grupos partían con un número muy parecido de matriculados. Bajo la rúbrica "no presentados" incluyo aquellos alumnos que no se presentaron a ningún examen durante el curso, es decir, ni siguieron el curso mediante evaluación continua, ni hicieron el examen final de la convocatoria de enero de 2019. El CIMA ha conseguido que la mayoría de estudiantes sigan las clases, en ambos grupos el absentismo es mucho menor al $50 \%$, a la vez que ha propiciado que más de la mitad de los matriculados superen la asignatura. Las diferencias en las notas medias también son muy significativas.

Con estos resultados no me quedó la menor duda en hacer extensiva la metodología a todos los grupos en los que imparto docencia en este curso y que dan contenido a esta comunicación.

Para contrastar la evolución del ciclo, el cuestionario antes reseñado pretende ser un inventario de todas las herramientas manejadas a lo largo del curso, elaborado a partir del planteamiento inicial y guiado a través de las propias preguntas. 
Si las respuestas están bien articuladas, deberían describir un proceso de inferencia estadística de manera que la respuesta a la primera pregunta plantearía la variable, la respuesta a la segunda describiría el proceso a seguir, la tercera y cuarta culminaría con la estimación y el contraste. La quinta pretende ir más allá, con un planteamiento, real también, pero con mayor complejidad.

\section{Conclusiones}

Este ciclo de mejora es el segundo intento, como señalé al principio, para implementar esta metodología en mi quehacer diario como docente. En gran medida usé las herramientas ya experimentadas en el curso anterior, pero entiendo este método como una forma viva de abordar la docencia, por lo que adquirió nuevas formas de relacionarnos y comunicarnos en el aula. Poco a poco voy venciendo los vicios adquiridos durante años de docencia siguiendo una metodología tradicional y cuyos resultados, como ya destaqué en la descripción del contexto, podemos calificar de desastrosos a la vista del alto número de repetidores y, sobre todo, de la dificultad que tienen los estudiantes para aprender los contenidos de una materia como la que nos ocupa, amén de las dificultades para comunicarnos en la transmisión de estos conocimientos.

Ya el pasado curso, la puesta en práctica de mi primer CIMA, en la medida que los alumnos manejaron la herramienta real y construyeron todo un proceso en el que iban necesitando herramientas para seguir adelante, pude constatar cómo se les abría las puertas del entendimiento, con lo que conceptos que antes parecían inaccesibles a la comprensión han llegado a sus mentes para quedarse, o al menos para ser almacenados en la carpeta de herramientas para aplicar, ahora disponen de herramientas útiles para explicar realidades, antes tenían un cajón de conceptos amontonados esperando a ser preguntados en un examen para salir de sus mentes sin retorno. 


\section{Bibliografía}

Bain, K. (2007). Lo que hacen los mejores profesores de universidad. Valencia: Universitat de Valencia.

Cano, A. (2018). Estadística Avanzada. 3a Ed. Red de Impresión.

Finkel, D. (2008). Dar clase con la boca cerrada. Valencia: Universitat de Valencia.

Manzano, V. (2012). La universidad comprometida. Bilbao. Hegoa.

Morin, E; Roger E y Domingo R. (2002). Educar en la era planetaria. El pensamiento complejo como método de aprendizaje en el error y la incertidumbre humana. Unesco.

Paenza, A. (2014). La puerta equivocada. Buenos Aires. Sudamericana.

Porlán, R. (2017). Enseñanza universitaria. Cómo mejorarla. Ediciones Morata, S. L.

http: / / www.magna-charta.org/resources/files/ the-magna-charta/spanish

Jornadas de Formación e Innovación Docente del Profesorado | № 2 (2019) Esta obra se distribuye con la licencia Creative Commons Reconocimiento-NoComercial-SinObraDerivada 4.0 Internacional (CC BY-NC-ND 4.0.) 